\title{
The New Relativistic Hydrodynamic Code for Numerical Simulation of Jet Evolution
}

\author{
Igor Kulikov*† \\ Institute of Computational Mathematics and Mathematical Geophysics SB RAS, \\ 6-th Lavrentyev avenue, Novosibirsk, Russian Federation \\ E-mail:
}

The program code for modeling the processes of special relativistic hydrodynamics is briefly described in the paper. The code is based on the author's library IPANEMA (I'm PArallel NEsted Mesh librAry) - the implementation of nested grids for architectures with shared and distributed memory. A brief description of a subset of the library for working with shared memory is presented in the paper. The results of numerical simulation of the central interaction of a relativistic jet with a dense gas cloud at various jet speeds are presented. A jet moves through a dense uniform gas and collides with a gas cloud, whose density exceeds ten times the background gas. The mechanism of dense clouds destruction when exposed to it by a relativistic jet is presented.

High Energy Phenomena in Relativistic Outflows VII - HEPRO VII

9-12 July 2019

Facultat de Fisica, Universitat de Barcelona, Spain

\footnotetext{
* Speaker.

${ }^{\dagger}$ This work was supported by Russian Science Foundation (project no. 18-11-00044).
} 


\section{Introduction}

The Relativistic Extragalactic Jets are important for energy and mass transfer from active core of galaxies into external environment. The mechanism of jets interaction with dense gas clouds is even more important task, since such interaction can significantly change flow direction and start the process of star formation in shocked clouds. Ultimately, it may be possible to explain the basic formation mechanism of the morphology of extragalactic radiojets [四].

A great number of works is devoted to the interaction of jets with the environment. Thus, the morphology of the nonrelativistic [0, [1, 田, 5] and the relativistic interaction of the jets with the

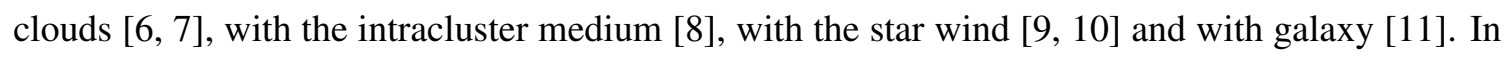
this paper, we consider an idealized central collision of a jet with a dense cloud at various collision speeds. Let us find out how much the collision speed affects the evolution of a dense cloud.

In the second section, the equations of special relativistic hydrodynamics are presented and the method for numerical solution is briefly described. The third section describes the capabilities of the IPANEMA author's library, which implements the functionality of nested grids for shared memory architectures. The results of code performance studies on IBM Power 9 processors are also presented in third section. The fourth section describes the formulation of the problem of jet interaction with dense gas cloud. The results of computational experiments are also presented in fourth section. The fifth section concludes.

\section{The numerical model}

To describe special relativistic hydrodynamics, we use the following primitive variables: $\rho$ is density, $\vec{v}$ is velocity vector, and $p$ is pressure. Let us introduce a special enthalpy $h$ defined by the equation (2.]):

$$
h=1+\frac{\gamma}{\gamma-1} \frac{p}{\rho}
$$

where $\gamma$ is the adiabatic index. The sound speed $c_{s}$ is determined by the equation (2.2):

$$
c_{s}^{2}=\gamma \frac{p}{\rho h} .
$$

In the present paper, we take the sound speed $c \equiv 1$. In this case the Lorenz factor, $\Gamma$, is defined by the equation (2.3]):

$$
\Gamma=\frac{1}{\sqrt{1-(v / c)^{2}}}=\frac{1}{\sqrt{1-v^{2}}} .
$$

Thus, the velocity modulus must not exceed unity.

Let us introduce the following conservative variables: $D=\Gamma \rho$ is relativistic density, $M_{j}=$ $\Gamma^{2} \rho h v_{j}$ is relativistic momentum, where $v_{j}$ are the components of the velocity vector $\vec{v}, j=1,2,3$, and $E=\Gamma^{2} \rho h-p$ is total relativistic energy. In this case, the special relativistic hydrodynamic equations in the form of conservation laws may be written as [ए2]:

$$
\frac{\partial D}{\partial t}+\frac{\partial\left(D v_{k}\right)}{\partial x_{k}}=0
$$




$$
\begin{gathered}
\frac{\partial M_{j}}{\partial t}+\frac{\partial\left(M_{j} v_{k}+p \delta_{j k}\right)}{\partial x_{k}}=0, \\
\frac{\partial E}{\partial t}+\frac{\partial(E+p) v_{k}}{\partial x_{k}}=0 .
\end{gathered}
$$

Note that in the numerical method the calculations are performed in the conservative variables $D$, $M_{j}$, and $E$ taking into account the nonlinear relationship between the conservative variables and the primitive ones, $\rho, v$, and $p$. A special recovery procedure for the primitive variables and some details of numerical method can be found in papers [13], [44, [15].

\section{The shared memory implementation}

The calculation scheme using nested meshes is shown in the figure (II) and described in details in [16]. The nested mesh is a structured object that was implemented in the IPANEMA author's

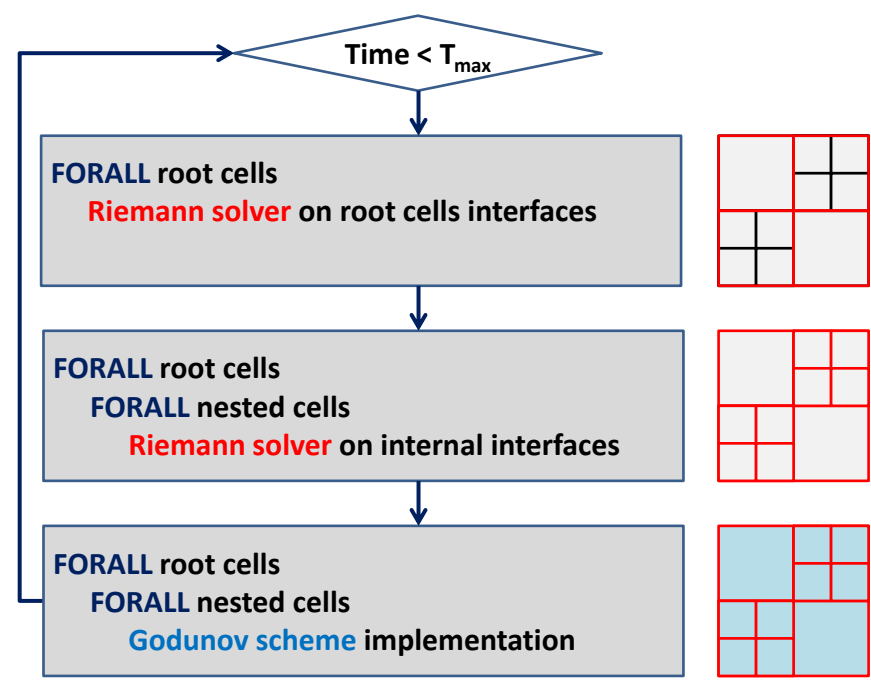

Figure 1: The Code Architecture

library using the BinaryNestedMesh $\mathrm{C}++$ class. The representation of nested grids using dynamic arrays is encapsulated into class. Note that each nested grid is cubic with a size equal to $2^{k}$, where $k>1$. In addition, two adjacent nested grids differ each other in size by no more than two. To represent a three-dimensional array, we use a one-dimensional array of the appropriate size and the inline private index function to determine the effective index. We describe the main methods of the class that allow you to organize calculations using nested grids:

1. The constructor of the BinaryNestedMesh class takes the size of the root grid as an input and creates a root grid in the form of an array of pointers to nested grids and an integer array to store the sizes of all nested grids - a portrait of nested grids.

2. The destructor of the BinaryNestedMesh class, which frees up all the allocated RAM.

3. The GetRootMeshSize method returns the size of the nested grid along each dimension. 
4. The SetNestedMeshSize method sets the size of the corresponding nested grid.

5. The GetNestedMeshSize method returns the size of the corresponding nested grid.

6. The CreatePortrait method allocates memory for all nested grids defined by the portrait. By the time this method is called, the dimensions of all nested grids must be set. By default, the dimensions of all nested grids are set to one.

7. The operator () - " brackets " returns the contents of the cell by the indices of the root and the corresponding nested grid.

8. The GetNeighbors method returns the number and contents of neighboring cells in a given direction. The method does not return more than four neighboring cells due to restrictions on the size ratio of neighboring nested cells.

Such set of methods makes it possible to implement the procedure of piecewise polynomial reconstruction of a solution. It means solution of the problem of disintegration of a gap and implementation of the Godunov scheme. A more detailed realization of the IPANEMA library and the BinaryNestedMesh class will be discussed in another publication.

A study of nested grids implementation using nested grids of the same size on IBM Power 9 processors showed that 60 -fold acceleration on 196 threads can be achieved. A 42x speed with 64 threads can be obtained when using only 25 percent of the nested meshes of large size. If an additional 10 percent of the nested grids is doubled, then 34-fold acceleration is achieved when using 48 threads. Such high acceleration rates became possible when using the two-level method of computing distribution using OpenMP tools: loop task + parallel for.

\section{The interacting of relativistic jet with dense cloud}

Let us simulate a galactic jet of density $\rho_{J}=10^{-1} \mathrm{~cm}^{-3}$ and radius $R_{J}=200$ parsec. The jet has the following properties:

1. The low relativistic case with Lorenz factor $\Gamma=5$.

2. The relativistic case with Lorenz factor $\Gamma=10$.

3. The ultra relativistic case with Lorenz factors $\Gamma=100$.

the temperature of the galactic atmosphere $T_{A}=10^{7} \mathrm{~K}$, and the density $\rho_{A}=1 \mathrm{~cm}^{-3}$. The adiabatic index $\gamma$ is taken to be equal to $5 / 3$. The density of dense cloud $\rho_{C}=10 \mathrm{~cm}^{-3}$. Figures (DI) show the results of a simulation of jet evolution in all cases. It can be seen from the figures, that in fact at any jet-speed the dense cloud is destroyed. With an increase in the Lorentz factor to $\Gamma=100$, the front of the dense cloud is destroyed more significantly. The spherical symmetry of the dense cloud is lost at the moment of collision. With a small Lorentz factor $\Gamma=5$, the impact region is limited and the rest of the cloud does not lose its symmetry. It can be seen from the figure (B]) that in the ultra-relativistic regime of jet is injected significantly into the cloud. The ultra-relativistic density contours significantly deviate from the circle in the region of the jet - cloud interaction boundary. In the case of a relativistic jet, such a deviation also exists, but less. In the case of a low relativistic 

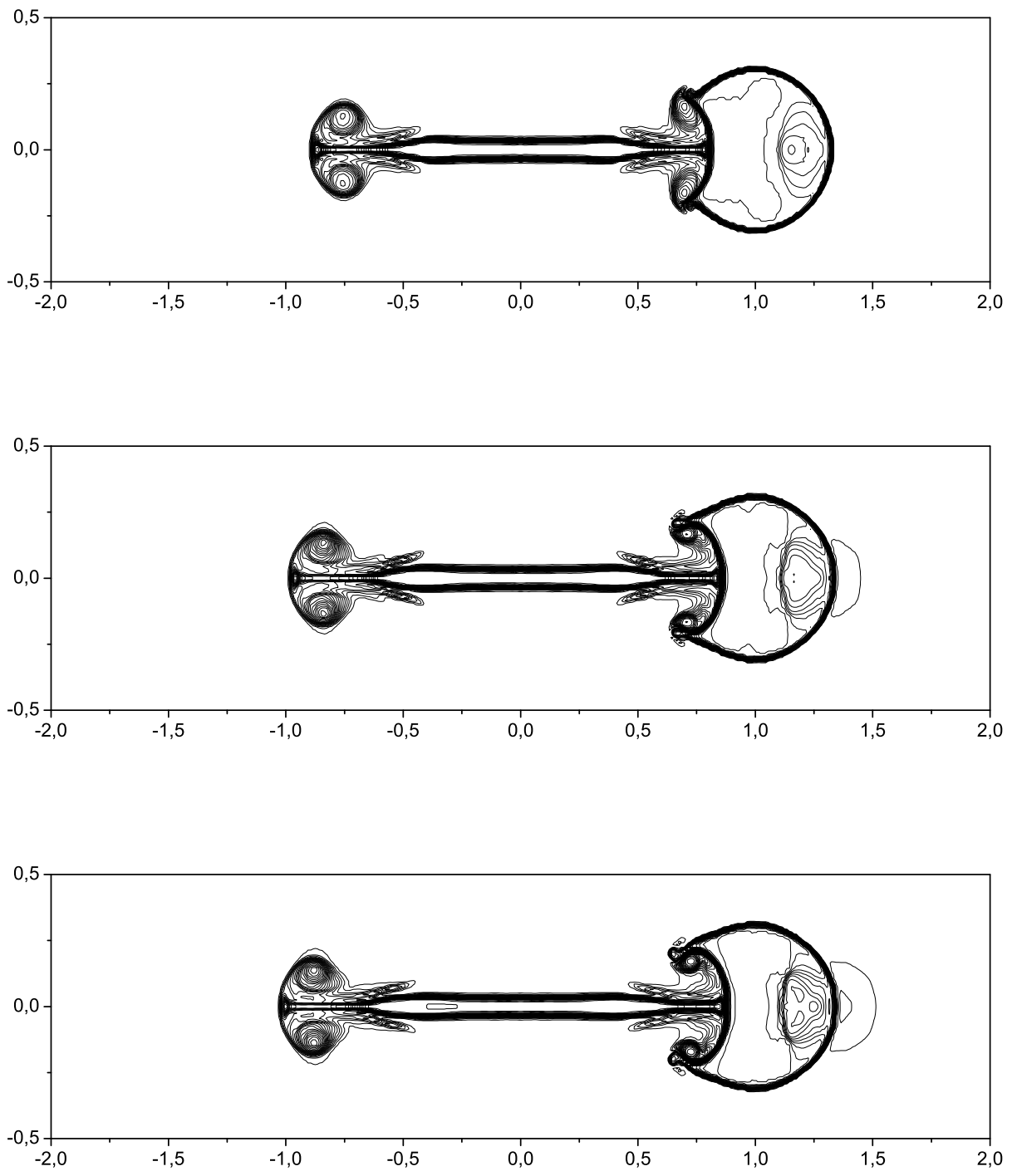

Figure 2: The density isolines for low relativistic (upper), relativistic (median), and ultra relativistic (down) cases.

jet, the density contours differ from the circle only in the impact region and do not change their spherical shape. From the simulation results, we can conclude that with an increase in the Lorentz factor, the inner part of the cloud is destroyed along with the shell.

\section{Conclusion}

The code for modeling the processes of special relativistic hydrodynamics is briefly described. To implement operations on working with nested grids, the author's IPANEMA library and the BinaryNestedMesh class were developed. The main class methods are presented in the paper. Acceleration of program implementation was achieved $34 \mathrm{x}$ up to $60 \mathrm{x}$ depending on grid uniformity. 


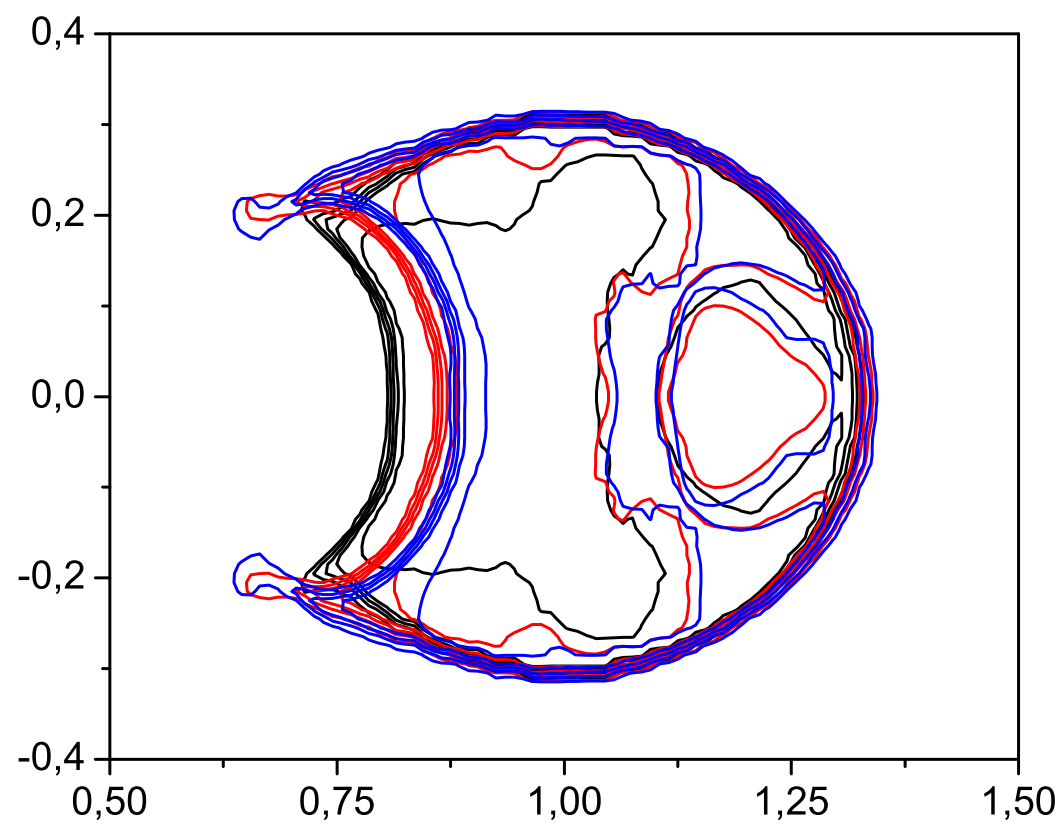

Figure 3: The compare of density isolines for low relativistic (black lines), relativistic (red lines), and ultra relativistic (blue lines) cases.

The model problem of the central interaction of a relativistic jet with a dense gas cloud at various jet speeds has been experimentally investigated. The destruction of dense clouds when exposed to it by a relativistic jet is shown.

\section{References}

[1] Fanaroff B.L., Riley J.M. The morphology of extragalactic radio sources of high and low luminosity // Monthly Notices of the Royal Astronomical Society. - 1974. - V. 167. - P. 31P-36P.

[2] de Gouveia Dal Pino E.M. Three-dimensional Simulations of Jet/Cloud Interactions: Structure and Kinematics of the Deflected Jets // The Astrophysical Journal. - 1999. - V. 526, I. 2. - P. 862-873.

[3] Higgins S.W., O’Brien T.J., Dunlop J.S. Structures produced by the collision of extragalactic jets with dense clouds // Monthly Notices of the Royal Astronomical Society. - 1999. - V. 309. - P. 273-286.

[4] Wang Z., Wiita P.J., Hooda J.S. Radio Jet Interactions with Massive Clouds // The Astrophysical Journal. - 2000. - V. 534. - P. 201-212.

[5] Saxton C.J., Bicknell G.V., Sutherland R.S., Midgley S. Interactions of jets with inhomogeneous cloudy media // Monthly Notices of the Royal Astronomical Society. - 2005. - V. 359 - P. 781-800.

[6] Choi E., Wiita P.J., Ryu D. Hydrodynamic Interactions of Relativistic Extragalactic Jets with Dense Clouds // The Astrophysical Journal. - 2007. - V. 655. - P. 769-780.

[7] Jeyakumar S. Interaction of radio jets with clouds in the ambient medium: Numerical simulations // Astronomische Nachrichten. - 2009. - V. 330. - P. 287-290. 
[8] Choi E. Relativistic hydrodynamic jets in the intracluster medium // Monthly Notices of the Royal Astronomical Society. - 2017. - V. 469. - P. 4148-4156.

[9] Bosch-Ramon V., Perucho M., Barkov M.V. Clouds and red giants interacting with the base of AGN jets // Astronomy \& Astrophysics. - 2012. - V. 539. - Article Number A69.

[10] Perucho M., Bosch-Ramon V., Barkov M.V. Impact of red giant/AGB winds on active galactic nucleus jet propagation // Astronomy \& Astrophysics. - 2017. - V. 606. - Article Number A40.

[11] Evans D.A., Fong W.-F., Hardcastle M.J. A Radio through X-Ray Study of the Jet/Companion-Galaxy Interaction in 3C 321 // The Astrophysical Journal. - 2008. - V. 675. - P. 1057-1066.

[12] Landau L.D., Lifshitz E.M. The Classical Theory of Fields // 4th Edition. - 1987. - 402 P.

[13] Kulikov I., Chernykh I., Tutukov A. A New Hydrodynamic Code with Explicit Vectorization Instructions Optimizations that Is Dedicated to the Numerical Simulation of Astrophysical Gas Flow. I. Numerical Method, Tests, and Model Problems // The Astrophysical Journal Supplement Series. 2019. - V. 243. - Article Number 4.

[14] Kulikov I., Chernykh I., Berendeev E., Karavaev D., Protasov V. Relativistic Hydrodynamics Modeling by means Adaptive Nested Mesh on IBM Power 9 // Communications in Computer and Information Science. - in print.

[15] Kulikov I., Chernykh I., Sapetina A., Prigarin V. A new MPI/OpenMP code for numerical modeling of relativistic hydrodynamics by means adaptive nested meshes // Journal of Physics: Conference Series. - 2019. - V. 1336 - in print.

[16] Kulikov I. The numerical modeling of the collapse of molecular cloud on adaptive nested mesh // Journal of Physics: Conference Series. - 2018. - V. 1103. - Article Number 012011. 